\title{
THE PROBLEM OF TRAUMA IN THE LOW BACK
}

\author{
By G. T. du Toit, F.R.C.S., Orthopaedic Surgeon.
}

$\mathbf{I}^{\mathrm{N}}$ NTEREST in injuries to the low back was manifested in the writing of Hippocrates 400 years B.C. It was recognised then that dislocations of vertebrae were fatal because of injury to the spinal cord. Lesser injuries were clinically diagnosed and successfully treated. Celsus, in the second century A.D., gave warning that inexpert treatment of dislocations of the spine could cause deterioration of the condition and death.

In A.D. 690 exploration of the injured spine through an incision was advocated to remove pressure of a bony fragment from the spinal cord. In Germany in 1600 several surgeons advocated exploration of the spine but not until 1762 was a successful operation quoted in France.

Astley Cooper in 1826 advised against manipulation of a dislocated spine. Benjamin Brodie manipulated fractures in the low back but thought it dangerous in the cervical spine. In England the attitude remained conservative and treatment was confined to rest in recumbency.

In 1843 Crowfoot treated a coachman who, while sitting on the box of his coach, struck his head against an archway which doubled him up, so producing a dorsolumbar kyphosis with paraplegia. With a belt under his arms fixed to the head of the bed and pelvic belt to which cords were attached over pulleys at the foot of the bed, the déormity gradually disappeared, paralysis recovered slowly and he eventually returned to work.

With the development of railways, the term "railway spine" covered a confused jumble of orthopaedic and functional sequelae. During the Crimean War it was felt that all cervical bony injury cases died in the field and dorsolumbar injuries predominated amongst those cases treated and studied.

Treatment was mainly conservative immobilisation on a waterbed with general nursing hygiene.

The plaster jacket immobilisation of tuberculous spines lead to the same vogue for fractures of the spine.

$\mathrm{X}$-ray developments in 1895 resulted in rapid progress in these studies. As a consequence, bony injuries assumed major importance and in the absence of neurological complications soft tissue injuries were largely forgotten or ignored.

In 1929, Davis drove home the point that the anterior common ligament was very strong and could prevent overreduction when the patient's feet were elevated and the spine extended to the limit.

In 1940, Watson Jones advocated the 2 table hyperextension cast. Bohler in 1935 showed the importance of efficient routine, accurate casting and early organised active exercises to maintain and regain muscle power. He stressed the value of segregation of similar cases, fostered the spirit of competition in group exercises and accentuated the influence of personal attention and practical psychotherapy in reducing the incidence of traumatic neuroses. On his work is based the present attitude of the physiotherapist towards this problem.

In 1929, Schmorl's pathological studies brought about intense interest in the subject of ligamentous injuries and intervertebral disc damage. In 1934, Mixter and Barr pioneered the study of herniated discs in relation to sciatica.

- Since then', the study of cervical discogenic pathology has advanced steadily.

Now we recognise a number of clinical pictures of painful spinal disabilities associated with mechanical pressure on nerve roots and spinal cord and it is becoming increasingly clear that many neuritic conditions such as brachial neuralgia and sciatica, as well as a number of previously obscure spinal cord diseases with damage to the tracts of the spinal cord are the conseguence of processes of degeneration of the spinal column due to combinations of ageing and traumatic incidents.

\section{STRUCTURAL CONSIDERATIONS:}

The dorsal spine is relatively rigid and restricted by costovertebral and costotransverse joints and intertransverse and associated ligaments, as well as by the configuration of the paravertebral joints and the narrow disc spaces.

In the low dorsal area of the false ribs mobility is great and, in the lumbar, even greater, where the discs increase relatively in thickness and the paravertebral joints are designed to give greater mobility in all directions. Longitudinal compression can injure the mobile lumbar curve in the position assumed under stress, e.g. flexion.

The lumbar vertebral body has a compact, bony shell, containing soft cancellous bone permeated by a blood sinus, the compact bony end plates being almost bloodless. Posteriorly the body is strengthened by articular processes and pedicles, so that it is compressible anteriorly rather than posteriorly.

The intervertebral disc with fibrocartilagenous end plates, elastic annulus fibrosus and a semifluid nucleus pulposus is the essential mechanism of the amphiarthrodial joint between vertebrae. These positive fluid pressure joints distribute the stress evenly over the bearing surfaces. The physico chemical processes keeping the disc in tension are not well understood.

The elastic annulus degenerates with increasing age. In the absence of weightbearing stress, two 'vertebral bodies are forced apart with a pressure of about 32 pounds. In the erect posture this pressure is greatly increased and in forward flexion the pressure reaches fantastic heights. The efficiency of this joint depends on internal fluid tension which intact cartilage plates and elastic annulus fibrosus are necessary. The disc structure itself is avascular. Variation in disc structure throughout life is considerable. Progressive change with advancing age is the key factor around which most spinal disabilities arise. These changes must be considered in relation to trauma.

In youth, movement of one vertebrae on the other is associated with accommodation of shape and redistribution of the semifluid core to adapt to movement in all directions.

In middle age, loss of fluid, loss of elasticity, fibrous replacement of elastic tissues causes loss of mobility of the fluid core of the disc and conseguently the elastic bending of the spinal column as a whole is lessened and displacement of vertebral bodies on each other becomes more of a sliding of one bone on the other. The annulus tends to break, the endplates tend to crack and fissure, and there is loss of height and loss of mobility of the spine on the whole. However, there is a phase after substantial loss of disc substance by prolapse, during which mobility at an isolated disc level may be increased temporarily before the scarring and settling together of vertebral bodies eliminates movement for practical purposes.

These changes may be more specifically analysed as follows:-

Nucleuspulposus defects occur with loss of mucoid semifluid structure and fibrous replacement. The demarcation between the nucleus and the annulus becomes less distinct. Cavities and splits develop in the disc substance. The residual semifluid centre may be displaced from its anatomical site and be incapable of resuming normal position. Herniation may occur through an endplate, through the annulus backwards or forwards.

The cartilage endplates sometimes have embryonic defects through which Schmorl's nodes push into the vertebral body. Trauma may produce gross fracture of the endplate and the body with escape of the nucleus into the body, but lesser injuries may only produce cracks in the endplate with delayed consezuences of the same type. As the disc material escapes it becomes scarred and walled off by peripheral 
sclerosis, confining the prolapse but by that time the disc has lost substance and has become narrowed.

The Annulus loses elasticity following degenerative changes associated with ageing and trauma. The' most common prolapse of the disc is through posterior developed defects in the annulus.

In osteoporosis from any cause the positive pressure in the disc causes expansion at the expense of bony structure producing biconcave vertebrae. Trauma can exaggerate this deformity. With age the dorsal curve increases and becomes less resistant to compressive force.

The mobile lumbar spine being subject to more wear and tear from movement, develops degenerative changes more rapidly and trauma is often the culminating factor giving rise to neurological damage and symptoms.

\section{SOFT TISSUE INJURIES}

The condition of "sprung back" described by Newman in 1952 , is now well recognised. It occurs often in women suffering from lumbosacral pain, which is a dull ache, a nagging pain and not often a sharp pain. The condition may come on after a fall, a blow or a strain while lifting a weight. Many of the patients are young housewives, nurses or young men doing occasional heavy manual work to which they are not accustomed. The pain is made worse by sitting in a slouching position, lifting a weight at arm's, length, bending or stooping with the knees straight, standing with a slight stoop as in washing, ironing, cooking, operating, gardening. Muscle spasm is not severe, active lumbar flexion is slightly restricted, other movements full. Neoruligical signs are absent. Straight leg raising is unimpaired until the pelvis starts rotating. Tenderness is in the midline between L5-SI or at the next higher level. Sometimes a definite depression can be felt when the spine is moderately flexed and contrasts markedly with the resistance of the intact supraspinous ligament at other levels. Tilting $X$-rays may show mechanical instability in lateral films taken in full flexion and extension.

Normally the supraspinous ligament acts as the main check ligament to overflexion. Its tension is greatest when the knees are extended. If the ligament ruptures the condition is a "sprung back". Greater violence would produce a compression fracture anteriorly. Occasionally the pars interarticularis breaks first to produce a traumatic spondylolysis. Rarely an articular facet may fracture. When the erector spinae muscles go into spasm, the torn structures often heal by close fibrous union, but the supraspinous ligament fails to heal adequately and may remain in an elongated and even an interrupted condition. The damaged paravertebral joint capsules would then also remain lax. The affected vertebrae are unstable. In other cases the scar remains painful under strain especially when the erector spinae becomes fatigued as in standing in a forward bent position.

Newman follows up this finding with that of more severe flexion strains causing damage to annulus and posterior longitudinal ligament and disc prolapse. So he explains the years of lumbago preceding prolapsed disc.

Strain of ligamentous attachments of vertebrae are common and no different to those in other sites such as medial collateral ligament strain in the knee from one injury or typical tennis elbow from multiple repeated stresses.

In more severe injuries it must be valid to assume partial rupture of muscles, ligaments, capsules and their attachments and, of course, small bony processes such as transverse processes which become avulsed by muscle action. These are similar to flake fractures of the great tuberosity of the humerus or an external malleolus in a severe sprain.

The problem is related to the severity of trauma and recognition of the necessity for healing to take place during rest and adequate splintage.

Inadequate appreciation of the need for prolonged rest in the severe sprain, accounts for the development of many chronic disabling back conditions. By contrast, the minor strains respond dramatically to heat, gentle massage, local aneasthetic infiltration, hydrocortone and activity.
That these injuries in the distribution of the posterior primary divisions of the spinal nerves can become trigger points of irritation with referred pain down the leg, is generally accepted and it is common practice to block these points with local aneasthetic with relief of referred pain, to distinguish this type of case from a genuine root compression causing sciatica.

The next problem in our rather empirical approach to clinical medicine, lands us in a quagmire, namely that of Congenital Anomalies. Ons is impressed by the recent analysis at the University of Helsinki by Bistrom who analysed 151 cases of men doing heavy manual work at all ages, and found Congenital Anomalies in 58; there were 13 cases of Spondylolisthesis; 8 of Spondylolysis; 2 of Spina bifida; numerous transitional vertebrae at L5 level; 25 cases of Sacralisation or lumbarisation; and 10 cases had a transversosacral joint on one side only.

None of this series had backache or any other symptoms. Such a finding justifies caution in the evaluation of back symptoms. Some loose thinking has crept into orthopaedic practice. There is a tendency to assert that once a workman in the third decade of life develops a clinical aggravation of a pre-existing Spondylolisthesis or pain in a transversosacral joint, that symptoms are likely to persist. In private practice, conservative treatment may restore the pre-accident state, but in industrial practice with the tardy response to treatment, the surgeon not infrequently succumbs to the desire to do something drastic, even operation, in his wish to finalise a case with intractible symptoms.

The surgical approach to those cases of Congenital Low Back Anomalies requiring treatment, is a complicated one. We cannot genuinely assert that our practice in this respect, at present, is logical and based on an adequate analysis of material.

Surgeons have preferences for different techniques and the standard answer for a problem such as Spondylolisthesis has not yet been agreed upon.

\section{DISC INJURIES}

The degenerate changes in discs have already been described. At all stages trauma may precipitate, accelerate or aggravate the condition. It appears to be well-accepted that degenerative changes appear at an early age but is not detachable clinically until the condition is advanced. Actual prolapse or herniation of a disc implies old or pronounced degeneration as a pre-existing condition in most cases. Massive acute prolapse after great violence may occur in young people under the age of twenty and is then usually associated with gross neurological signs even as extensive as paraplegia. Pathological studies appear to indicate that in the upper spine the degenerative changes are diffuse but in the lower 2 spaces the weakness develops mainly posteriorly. Apparently increased pressure on the posterior common ligament causes pain. Disc degeneration is a much more common cause of low back pain than lumbosacral anomalies.

Instability or sliding motion of vertebrae bodies is an early sign of disc degeneration and loss of fulcrum function.

The differential diagnosis of prolapsed disc includes conditions such as ankylosing spondylitis, osteomyelitis of spine, neoplasm of any of the related structures, chronic infections such as tuberculosis and a large variety of soft tissue strains in the sprained back category.

The problem of the coincidental occurrence of a Vertebral body fracture and a traumatic prolapse of a disc at a different level, occurs occasionally. It is clear that the responsibility of diagnosis in these cases is not always sufficiently appreciated.

The indiscretion of submitting such spines to treatment by physical methods is obvious. How intermittent traction, or head-halter traction or manipulation can have any permanent beneficial effect on pathology of this type, is beyond understanding.

\section{ACCESSORY METHODS OF INVESTIGATION}

Myeolgraphy has inadequacies for diagnostic purposes under some conditions and laterally placed prolapsed discs 
do not necessarily indent the column of pantopaque. In certain individuals the neural canal is narrower than usual. A negative or inconclusive myelogram need not be a deterrent to surgery in the face of clear clinical findings. Patients, therapists, and doctors sometimes attach undue value to a negative myelogram.

Discography has certainly gained a place in preoperative diagnosis and will sometimes show a degenerative disc with diffused and dissipated opaque pyelosil at the L4-5 level, and a normal disc at the level L5-S1, when the reverse is expected on clinical grounds. When a tumour is suspected, a surgeon will usually prefer myelographic investigation. There are technical difficulties in the interpretation of discograms but greater experience is being acquired in this field.

Surgical treatment: True perspective towards operation is developing as a result of intensive study and statistical research. Surgical treatment is absolutely indicated only for sudden severe neurological disturbance. Even the worst cases, low back pain and sciatica, due to prolapsed disc, are amenable to conservative methods in any particular attack. Recurrent severe incapacitation in relation to work and way of life is the more usual justification for operation. Much harm is still done in disc surgery under circumstances where technical skill or facilities are inadequate. But this statement may be true of any field of surgery.

\section{PRE-EMPLOYMENT EXAMINATION}

For many years it has been common experience in industrial medical practice that one of the most costly disabilities, from the point of view of loss of time and permanent disablement, is low back strain and its consequences.

In recent years pre-employment radiological surveys of the lumbosacral spine has been performed in some industries, especially in America.

Examination of this type reduces losses from the compensation point of view just as aptitude testing has become an accepted technique in placement of employment. By avoiding the placing in wrong employment, a weak spine can be saved from crippling strain. It is surely better for the individual with an architecturally weak spine to seek less arduous employment than to attempt work beyond his powers and to become disabled to a severe degree. Even in the event of a man being accepted for employment with a defective back, the previous record of pre-existing defect would be valuable.

It is not claimed that this procedure is a panacea for the problem of compensation claims due to injury or strain of the low back. A study performed by the American Locomotive Company, New York, on a series of 4,654 cases, found $25.44 \%$ which showed defects which the authors of the paper regarded as of actual or potential clinical significance. About $10 \%$ of applicants were rejected for employment because of gross defects. The tendency is to adopt even more rigid standards of acceptance. So many cases of back injury fall within the field of Workmen's Compensation that it is of interest to discover that the Compensation Act as it functions in New York State, is more of an economic detriment than a boon to the injured workman.

In 160 cases which collected compensation, the review showed that $20 \%$ were consistent with degeneration of $\mathrm{a}$ lumbar disc while in $80 \%$ an anxiety state was revealed by the presence of multiple, unrelated complaints, failure to establish a firm clinical diagnosis and psychiatric findings of intrinsic factors in the patient's personality, extrinsic factors associated with litigation, all of which resulted in failure to achieve rehabilitation up to the time of the assessment of the claim

Our main hope appears to be in the field of reducing the number of cases where we are dependant upon subjective symptoms by greater accuracy in objective evaluation. It is hoped that in this field discography will assist in reducing guesswork and increasing the number of cases in which objective analysis is practicable.

A positive, constructive, optimistic attitude is required for primary rehabilitation of the man with the injured back. More workmen can be restored to activity, industrial man- power can be saved and the ascent of Compensation can be reduced.

Fractures: Touching briefly on the problem of compression fractures, it should be recorded that minor and moderate stable compression fractures are no longer immobilised in plaster casts but the probability exists that cases requiring enough rest to consolidate and scar up, may be mobilised too soon by those who over-enthusiastically try to prove their dislike of hyper-extension casts.

Inadequate $\mathrm{X}$-rays may fail to demonstrate disruption of endplates and herniation of discs into the vertebral body and lead to inadequate immobilisation, and a crop of late, painful, unstable backs with subluxated paravertebral joints may be anticipated.

Disability evaluation: Comparatively few surgeons have the opportunity of seeing large numbers of injured backs, especially since the entire profession is now participating in this field of medical service. It is one of the doubtful benefits of the "free choice of doctor" principle. Much attention in this field of surgery is being devoted to the evaluation of the effectiveness of the patient's doctor in this role.

In 1955, Kessler reviewed the work on 23,000 cases of low back pain assessed under the Workmen's Compensation in New York in one year, in 1952. Cases involving soft tissue only averaged 46 weeks of temporary total disability, as contrasted with six weeks in non-industrial cases. In cases of bone injury the temporary total disability period averaged 232 weeks, i.e. $4 \frac{1}{2}$ years. In non-industrial cases the average was 6 months. It is appalling to think that the injured workman was off ten times as long as the average private case.

\section{CONCLUSION}

In this country the evils of compensitis have only appeared occasionally.

The impact of unfair claims on accident funds and insurance carriers has been comparatively slight.

There is need, however, for firm action to back injuries to prevent such eventualities.

The isolated practitioner who sees an occasional case of back injury, is handicapped in his attempts at getting such a man back to normal activity in minimum time.

Fortunately, there are developments in a number of large centres in South Africa, which indicate a more realistic and effective approach to the problem of the injured person.

A team of doctor, physiotherapist, occupational therapist, social worker, placement officer and others, can deal much more efficiently with these problems.

The establishment of the Rehabilitation Association for Injured Workmen by the Department of Labour was a progressive move. The pilot scheme, the Workmen's Rehabilitation Centre, is a small beginning of an important social service in this community.

As the chronic crippling diseases and poliomyelitis are more effectively combated, the accent will increasingly shift to traumatic work.

\section{GENERAL}

Miss E. Botting, the Journal Editor, is at present overseas. We would like to wish her a very happy stay, and wish Mrs. Botting a speedy recovery from her recent operation.

Miss J. Blair, the Society Chairman, has recently returned from a month's tour of the Rhodesias.

Miss M. Hinz, from Pretoria, and Mrs. H. B. Hope, from England, are welcomed on to the Physiotherapy Staff of the University of the Witwatersrand.

Mrs. J. Jooste and Miss J. Crewe Brown, both from Pretoria, are welcomed on to the staff of the Pretoria Hospital School of Physiotherapy.

We wish good luck to Miss M. Morgan and Miss M. E. Rehfeld, who have left Pretoria for overseas, the latter to take her teaching diploma.

Miss J. Muller, an ex Pretoria student who has been working at Stoke Mandeville Hospital, is now proceeding to Canada for further experience. 